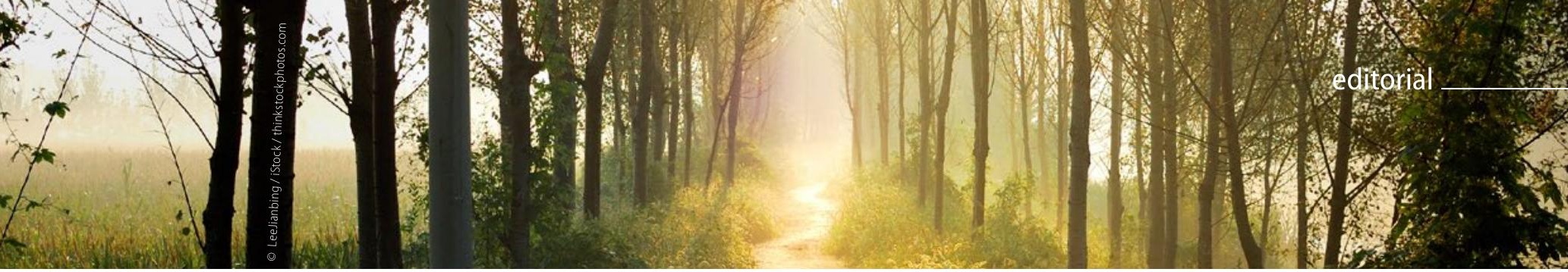

\title{
Was kommt auf uns zu?
}

In diesem Jahr sind fünf wichtige Projekte, teils Gesetzesregelungen, teils Reformen im ärztlichen Bereich „im Werden“. Das Krankenhausstrukturgesetz wird in Kürze, wohl aber erst im neuen Jahr, verabschiedet werden mit vielen Unklarheiten, wie sich das Gesetz dann in der Folge auf die Krankenhäuser auswirken wird. Eine ganze Reihe von Punkten sind umstritten.

Die Novellierung des Wissenschaftszeitvertragsgesetzes wird ebenfalls bearbeitet. Ob dort der Situation der ärztlichen Wissenschaftlerinnen und Wissenschaftler Rechnung getragen wird, ist eher zweifelhaft. Denn ärztliche Wissenschaftler haben ja die Sondersituation, dass Sie neben der Qualifizierung als Wissenschaftler auch noch parallel ihre ärztliche Weiterbildung zum Facharzt vorantreiben müssen. Hier müssten also längere, an die Weiterbildung angepasste Vertragslaufzeiten die Folge sein.

Der weitere große Wurf ist der Masterplan Medizinstudium 2020. Das Medizinstudium wird überdacht und reformiert werden. Hier enthält der Koalitionsvertrag der Bundesregierung die Regelung: „Zur Förderung der Praxisnähe und zur Stärkung der Allgemeinmedizin im Studium soll in einer Konferenz der Gesundheits- und Wissenschaftsminister von Bund und Ländern ein Masterplan Medizinstudium 2020 entwickelt werden. Die Förderung der Weiterbildung in der Allgemeinmedizin soll dazu um $50 \%$ erhöht werden. Zudem ist vorgesehen, die ärztliche Weiterbildung aller grundversorgenden Fachgebiete in ambulanten Einrichtungen zu fördern. Das Bundesministerium für Gesundheit unter Herrn Minister Gröhe und das Bundesministerium für Bildung und Forschung unter Frau Prof. Wanka haben sich verständigt, bis Ende 2015 Handlungskonzepte zu folgenden Themenblöcken zu erarbeiten:

- Stärkung der Allgemeinmedizin im Studium

- Förderung der Praxisnähe

- Zielgerichtete Auswahl der Studienplatzbewerber

Diese Handlungskonzepte sollen dann im Jahr 2016 Gegenstand der Beratungen in einer Konferenz der Gesundheits- und Wissenschaftsminister von Bund und Ländern sein.

Die Novellierung der GOÄ lässt auf sich warten und man wird sehen, wie diese dann angenommen werden wird.

Und nebenbei erfolgt auch noch die Überarbeitung der Musterweiterbildungsordnung auf Ebene der Bundesärztekammer und teils in Arbeitsgruppen mit Delegierten der Länderkammern, das heißt, das, was die Ärztinnen und Ärzte in Weiterbildung für die jeweiligen Fachgebiete können, bzw. beherrschen müssen. Das ist natürlich ein Bereich, den wir als Dermatologinnen und Dermatologen auch sehr gut begleiten und im Auge behalten sollten. Ich war bei der Bundesärztekammer zur Überarbeitung des Bereichs Dermatologie eingeladen und konnte mich so vom Stand der Dinge und den bisherigen Eingaben/Vorschlägen der Deutschen Dermatologischen

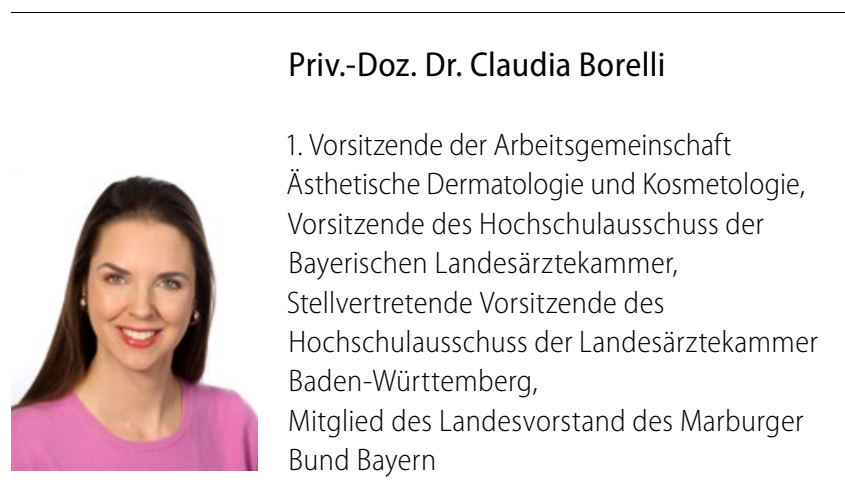

Gesellschaft, des Berufsverbands und anderen Fachgruppen informieren. Hier wird das Ergebnis allerdings sicher noch ein bis zwei Jahre auf sich warten lassen, bis es dann im Jahr 2017, aber eher 2018 auf dem Deutschen Ärztetag abgestimmt und verabschiedet werden wird.

Wir Dermatologinnen und Dermatologen sollten nicht nachlassen, solche Gesetzesänderungen oder Kammerregelungen zu begleiten und unsere Vorstellungen einzubringen. Nur durch Engagement in den Gremien, wie dem Berufsverband, den Länderkammern und anderen Verbänden kann es gelingen, Interessen zu bündeln und sich mit guten Argumenten durchzusetzen. Die Arbeitsgemeinschaft Ästhetische Dermatologie und Kosmetologie (ADK) und die Deutsche Gesellschaft für Dermatochirurgie (DGDC) haben zusammen ihre Sichtweise hinsichtlich der Weiterbildungsordnung für das Fach Dermatologie besprochen. Die Interaktion mit anderen Verbänden ist hier ein Muss und wichtig.

Wir Dermatologinnen und Dermatologen sollten in unserem Engagement für die kommenden Reformen nicht nachlassen und unbedingt miteinander das Gespräch suchen. Mein Appell an Sie: Engagieren Sie sich in der Politik, in Gewerkschaften (Marburger Bund, u.a.), in den Fachverbänden (ADK, Berufsverband, DGDC u.v.m.) und Gremien wie den Ärztekammern und auf regionaler Ebene. Nur so lassen sich unsere Interessen für das Fach Dermatologie, für die Medizin in Deutschland und für unsere Patienten sinnvoll durchsetzen.

\section{Herzlichst

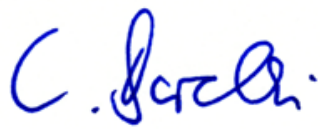

